\title{
Some properties of the Kermack-McKendrick epidemic model with fractional derivative and nonlinear incidence
}

\author{
Emile Franc Doungmo Goufo ${ }^{*}$, Riëtte Maritz and Justin Munganga
}

\section{"Correspondence:}

dgoufef@unisa.ac.za

Department of Mathematical

Sciences, University of South Africa,

Florida, 0003, South Africa

\begin{abstract}
Kermack-McKendrick epidemic model is considered as the basis from which many other compartmental models were developed. But the development of fractional calculus applied to mathematical epidemiology is still ongoing and relatively recent. We provide, in this article, some interesting and useful properties of the Kermack-McKendrick epidemic model with nonlinear incidence and fractional derivative order in the sense of Caputo. In the process, we used the generalized mean value theorem (Odibat and Shawagfeh in Appl. Math. Comput. 186:286-293, 2007) extended to fractional calculus to conclude some of the properties. A model of the Kermack-McKendrick with zero immunity is also investigated, where we study the existence of equilibrium points in terms of the nonlinear incidence function. We also establish the condition for the disease free equilibrium to be asymptotically stable and provide the expression of the basic reproduction number. Finally, numerical simulations of the monotonic behavior of the infection are provided for different values of the fractional derivative order $\alpha(0 \leq \alpha<1)$. Comparing to the model with first-order derivative, there is a similar evolution for close values of $\alpha$. The results obtained may help to analyze more complex fractional epidemic models.
\end{abstract}

Keywords: fractional epidemic model; Kermack-McKendrick model; nonlinear incidence; existence of solutions; wellposedness

\section{Introduction and important facts}

Considered as one of the first compartmental models, Kermack-McKendrick epidemic model was developed in the late 1920s with the pioneering work of Kermack and McKendrick $[1,2]$. The model is described as the SIR model for the spread of disease, which consists of a system of three ordinary differential equations characterizing the changes in the number of susceptible (S), infected (I), and recovered (R) individuals in a given population. The model is a good one for many infectious diseases, despite its simplicity. Ever since, numerous and more complex compartmental mathematical models have been developed. For instance in biology, modeling is particularly useful in studying organs like the lungs, heart, intestinal edema and cancer, etc. Almost all these models take their source on Kermack-McKendrick's model and serve to help gain insights into the transmission and control mechanisms of diseases like HIV, TB, malaria and their interactions with others. Then most of the works done on modeling the dynamics of epidemiological diseases 
have been limited only to models based on (a system of) classical first-order differential equations.

However, there is a growing interest in applying fractional calculus to mathematical epidemiology since it has turned out recently that many phenomena in different fields, including sciences, engineering, and technology, can be described very successfully by the models using fractional-order differential equations. As an example, the concept of a fractional Laplacian operator in the theory of Lévy flights [3] is a typical application of fractional derivatives. It leads to the theory of sub- and super-diffusion, which is well applicable in reaction-diffusion systems. In the field of mathematical epidemiology, especially the super-diffusion case is of interest since it describes a more realistic spreading than normal diffusion on regular lattices. Moreover, it has recently been noted [4] that some epidemic models based on dynamics with first-order derivative were unable to reproduce the statistical data collected in a real outbreak of some disease with enough degree of accuracy. Another example is the application of half-order derivatives and integrals that proved to be more useful and reliable for the formulation of certain electrochemical problems [5-7] than the classical models with derivative of order one. Accordingly, a number of works [8-14] have successfully generalized, in various ways, classical derivatives of integer order to derivatives of fractional order.

Therefore, following the same idea as above, we analyze and provide some interesting properties of the Kermack-McKendrick epidemic model with nonlinear incidence and fractional derivative order less than one. Therefore, we generalize the classical KermackMcKendrick model with the standard mass balance incidence.

\section{Model construction}

In this model, a population of size $N(t)$ is divided into different classes, disjoint and based on their disease status. At time $t, S=S(t)$ is the part of population representing individuals susceptible to a disease, $I=I(t)$ is the part of population representing infectious individuals, $R=R(t)$ is the part representing individuals that recovered from the disease. We will need the following hypotheses about the transmission process of the infectious disease and its host population.

- The transfer rates from a compartment to another is supposed to be proportional to the population size of the compartment. For example, the transfer rate from $S$ to $I$, the (nonlinear) incidence rate can be written as $\beta g(I) S(t)$, where $\beta$ is some rate constant and the function $g$ characterizing the nonlinearity is assumed to be at least $C^{3}\left(0, N_{0}\right]$ with $g(0)=0$ and $g(I)>0$ for $0<I \leq N_{0}$, with $N_{0}=N(0)$ the initial total population. Note that the classical mass balance incidence has $g(I) \equiv I$ and $\beta$ is called the transmission coefficient.

- The ensemble of individuals in the host population is well mixed and homogeneous so that the law of mass action holds: the number of contacts between hosts from different compartments depends only on the number of hosts in each compartment. For instance, the recovery rate, that is, the number of individuals recovering from the disease per unit time, can be expressed as $\mu I$, with $\mu$ the recovering rate.

- The transmission mode is supposed to be horizontal and happens through direct contact between hosts.

- There is no latent period and all the infected individual hosts become infectious following an infection. 


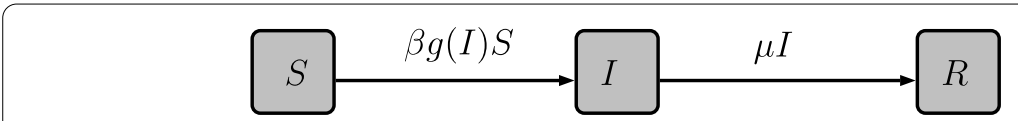

Figure 1 Transfer diagram for the Kermack-McKendrick model.

- There is no reinfection or loss of immunity and then no transfer from the compartment $R$ back to $S$.

Although they are restrictive, these assumptions proved to be very realistic for the diffusion of certain diseases, like for instance within the population of children in a child daycare or student population on a campus, where mixing occurs mainly through playing together in groups or going to classes, cafeteria, and so on. In addition to the above assumptions, we assume that there is no recruitment of new susceptibles and no removal from any compartments, therefore, the transfer diagram is given as in Figure 1 and the model reads

$$
\left\{\begin{array}{l}
D_{t}^{\alpha} S=-\beta g(I) S \\
D_{t}^{\alpha} I=\beta g(I) S-\gamma I, \\
D_{t}^{\alpha} R=\gamma I, \quad t>0,
\end{array}\right.
$$

subject to the initial condition

$$
S(0)=S_{0}, \quad I(0)=I_{0}, \quad R(0)=R_{0}, \quad\left(S_{0}, I_{0}, R_{0}\right) \in \mathbb{R}_{+}^{3} .
$$

Here,

$$
D_{t}^{\alpha} U(t)=\frac{\partial^{\alpha}}{\partial t^{\alpha}} U(t)=\frac{1}{\Gamma(1-\alpha)} \int_{0}^{t}(t-r)^{-\alpha} \frac{\partial}{\partial r} U(r) d r,
$$

with $0 \leq \alpha<1$ being the fractional derivative of the function $U(t)$ in the sense of Caputo [15], with $\Gamma$ the gamma function.

\section{Properties of the model}

\section{Property i: total population}

Consider $N(t)=S(t)+I(t)+R(t)$ and $N(0)=N_{0}=S_{0}+I_{0}+R_{0}$. Then the addition of all three equations in (1) yields

$$
D_{t}^{\alpha} N(t)=0 \quad \text { for all } t>0
$$

Taking the Laplace transform of both sides of this equation yields

$$
s^{\alpha} \tilde{N}(s)-s^{\alpha-1} N(0)=0,
$$

where $\tilde{N}(s)$ is the Laplace transform $\mathcal{L}(N(t), s)$. Taking the inverse Laplace transform of both sides of (4) gives

$$
N(t)=N_{0} \quad \text { for all } t>0
$$

and therefore, the model (1) assumes that the total population is constant all the time. 


\section{Property ii: wellposedness}

If the initial condition is nonnegative then the corresponding solution $(S(t), I(t), R(t))$ of the fractional model (1) is nonnegative all the time $t>0$.

To show it, we can investigate the direction of the vector field $(-\beta g(I) S, \beta g(I) S-\gamma I, \gamma I)$ on each coordinate plane and see whether the vector field points to the interior of $\mathbb{R}_{+}^{3}=$ $\left\{(S, I, R) \in \mathbb{R}^{3}: S \geq 0, I \geq 0, R \geq 0\right\}$ or is tangent to the coordinate plane. Indeed:

(a) On the coordinate plane $I R$, we have $S=0$ and

$$
D_{t}^{\alpha} S_{\mid S=0}=0
$$

(b) The same way, on the coordinate plane $S R$, we have $I=0$ and

$$
D_{t}^{\alpha} I_{\mid I=0}=\beta g(0) S=0 .
$$

(c) Finally, on the coordinate plane $S I$, we have $R=0$ and

$$
D_{t}^{\alpha} R_{\mid R=0}=\gamma I \geq 0
$$

To conclude the property, we need the generalized mean value theorem proved in [16] and stated as follows.

Theorem 3.1 Let the function $U \in C\left[t_{1}, t_{2}\right]$ and its fractional derivative $D_{t}^{\alpha} U \in C\left(t_{1}, t_{2}\right]$ for $0 \leq \alpha<1$, and $t_{1}, t_{2} \in \mathbb{R}$ then we have

$$
U(t)=U\left(t_{1}\right)+\frac{1}{\Gamma(\alpha)} D_{t}^{\alpha} U(\tau)\left(t-t_{1}\right)^{\alpha} \quad \text { for all } t \in\left(t_{1}, t_{2}\right]
$$

where $0 \leq \tau<t$.

Thus, considering the interval $\left[0, t_{2}\right]$ for any $t_{2}>0$, this theorem implies that the function $U:\left[0, t_{2}\right] \rightarrow \mathbb{R}^{+}$is nonincreasing on $\left[0, t_{2}\right]$ if $D_{t}^{\alpha} U(t) \leq 0$ for all $t \in\left(0, t_{2}\right)$ and nondecreasing on $\left[0, t_{2}\right]$ if $D_{t}^{\alpha} U(t) \geq 0$ for all $t \in\left(0, t_{2}\right)$. Then, from the points (a)-(c) above, the vector field on each coordinate plane is either tangent to the coordinate plane or and points to the interior of $\mathbb{R}_{+}^{3}$.

\section{Property iii: extension of solutions on $(-\infty,+\infty)$}

The fundamental theory of differential equations (with integer or noninteger derivative order) tells us that their bounded solutions can be extended for all time $t \in \mathbb{R}$. From Properties $\mathrm{i}$ and ii we have at least $N_{0} \geq S \geq 0, N_{0} \geq I \geq 0$, and $N_{0} \geq R \geq 0$, meaning that solutions $(S(t) ; I(t) ; R(t))$ are bounded in their maximal interval of existence. Thus, solutions to the fractional model (1) exist for $t \in(-\infty,+\infty)$.

\section{Property iv: asymptotic behavior of solutions}

We aim to show the existence of

$$
\lim _{t \rightarrow \infty} S(t)=S_{\infty}, \quad \lim _{t \rightarrow \infty} I(t)=I_{\infty}, \quad \lim _{t \rightarrow \infty} R(t)=R_{\infty}
$$


Indeed, the first line of (1) yields

$$
D_{t}^{\alpha} S=-\beta g(I) S \leq 0, \quad \text { since } g(I) \geq 0 .
$$

Thus, from Theorem 3.1, $S(t)$ is nonincreasing (decreasing) on its domain of existence and since $0 \leq S(t)$ for all $t \in(-\infty,+\infty)$, we conclude that $S_{\infty}$ exists and $0 \leq \lim _{t \rightarrow \infty} S(t)=S_{\infty}$. In the same way, the third line of (1) gives

$$
D_{t}^{\alpha} R=\gamma I \geq 0
$$

and from Theorem 3.1, $R(t)$ is nondecreasing (increasing) on its domain of existence and since $N_{0} \geq R(t)$ for all $t \in(-\infty,+\infty)$, we conclude that $R_{\infty}$ exists and $N_{0} \geq \lim _{t \rightarrow \infty} S(t)=$ $R_{\infty}$

Finally, the existence of $I_{\infty}\left(=N_{0}-S_{\infty}-R_{\infty}\right) \geq 0$ is therefore obvious since $S_{\infty}$ and $R_{\infty}$ exist.

\section{Kermack-McKendrick fractional model for disease with zero immunity and nonlinear incidence}

\subsection{Existence of equilibrium points when $0 \leq \alpha<1$}

The model (1) with zero immunity means any recovered person becomes immediately susceptible, leading to the diagram in Figure 2 and being expressed by the system

$$
\left\{\begin{array}{l}
D_{t}^{\alpha} S=b-\beta g(I) S+\gamma I-d S \\
D_{t}^{\alpha} I=\beta g(I) S-\gamma I-d I
\end{array}\right.
$$

subject to the initial condition

$$
S(0)=S_{0}, \quad I(0)=I_{0}, \quad\left(S_{0}, I_{0}\right) \in \mathbb{R}_{+}^{2},
$$

where $b$ and $d$ are, respectively, the birth and death rate and assumed to be constant. We evaluate the equilibrium points of the model (6) by letting

$$
\left\{\begin{array}{l}
D_{t}^{\gamma} S=0 \\
D_{t}^{\gamma} E=0
\end{array}\right.
$$

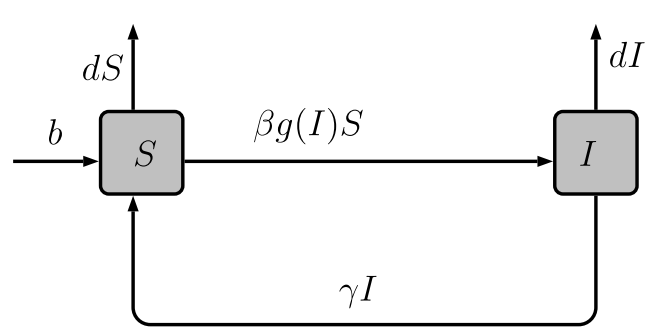

Figure 2 Transfer diagram for the Kermack-McKendrick model with zero immunity. 
which yields the disease free equilibrium (DFE) given by $x^{o}=\left(\frac{b}{d}, 0\right)$. The endemic equilibrium point if it exists is given by $x^{e}=(\bar{S}, \bar{I})$ where $\bar{I}$ satisfies the equation

$$
\frac{d(\gamma+d)}{b \beta}=\frac{g(I)}{I}\left(1-\frac{I}{\frac{b}{d}}\right) \equiv h(I)
$$

and $\bar{S}=\frac{b}{d}-\bar{I}$.

Recall [17] that $\frac{b}{d}$ is the maximum possible value of $\bar{I}$ and in the classical mass action incidence, $\frac{b \beta}{d(\gamma+d)}$ is called the contact reproduction number. Note that the number of solutions of (9) depends on the nonlinear incidence function $g(I), \lim _{I \rightarrow 0} \frac{g(I)}{I} \equiv h(0)$ and the sign of $h^{\prime}(I)$.

Theorem 4.1 The model (6) has no endemic equilibrium point if $h(0) \leq \frac{d(\gamma+d)}{b \beta}$ and $h^{\prime}(I)<$ 0 for all $I \in\left(0, \frac{b}{d}\right)$ and has one endemic equilibrium point if $h(0)>\frac{d(\gamma+d)}{b \beta}$ and $h^{\prime}(I)<0$ for all $I \in\left(0, \frac{b}{d}\right)$.

Proof The proof of this theorem follows the same approach as [18, Theorem 3.1], where we can substitute $H$ by $\frac{b}{d}$ and $\sigma$ by $\frac{b \beta}{d(\gamma+d)}$.

\subsection{Stability of the DFE point $x^{0}$ when $0 \leq \alpha<1$}

To study the stability of the DFE point $x^{o}=\left(\frac{b}{d}, 0\right)$ of the fractional model, we evaluated at the DFE the Jacobian matrix $J\left(x^{o}\right)$ for the system given in (6), which reads as follows:

$$
J\left(x^{o}\right)=D f\left(x^{o}\right)=\left[\begin{array}{cc}
-d & \frac{-\beta g^{\prime}(0) b}{d}+\gamma \\
0 & \frac{\beta g^{\prime}(0) b}{d}-(\gamma+d)
\end{array}\right] \text {. }
$$

Theorem 4.2 Consider the nonlinear incidence function $g$. The disease free equilibrium of the system (6) is asymptotically stable if

$$
\frac{\beta g^{\prime}(0) b}{d(\gamma+d)}<1
$$

Proof We know, see [19], that the disease free equilibrium for a fractional model of type (6) is asymptotically stable if all of the eigenvalues, $\lambda_{1}$ and $\lambda_{2}$, of $J\left(x^{\circ}\right)$ satisfy the following constraint:

$$
\alpha \frac{\pi}{2}<\left|\arg \lambda_{i}\right|
$$

for $i=1,2$ and $0 \leq \alpha<1$. The eigenvalues of $J\left(x^{o}\right)$ are

$$
\lambda_{1}=-d
$$

and

$$
\lambda_{2}=\frac{\beta g^{\prime}(0) b}{d}-(\gamma+d)
$$

It is obvious that $\lambda_{1}$ satisfies (11) and $\lambda_{2}$ satisfies (11) if $\frac{\beta g^{\prime}(0) b}{d(\gamma+d)}<1$, which concludes the proof. 
For the fractional model (6), the quantity $\frac{\beta g^{\prime}(0) b}{d(\gamma+d)}$ is usually referred to as the basic reproduction number denoted by $\mathcal{R}_{0}$ and defined as the number of secondary cases that one case will produce in a completely susceptible population. The biological interpretation of $\mathcal{R}_{0}$ is that if $\mathcal{R}_{0}$ exceeds one, then the epidemic will occur, but if it is less than one, then the infection dies out.

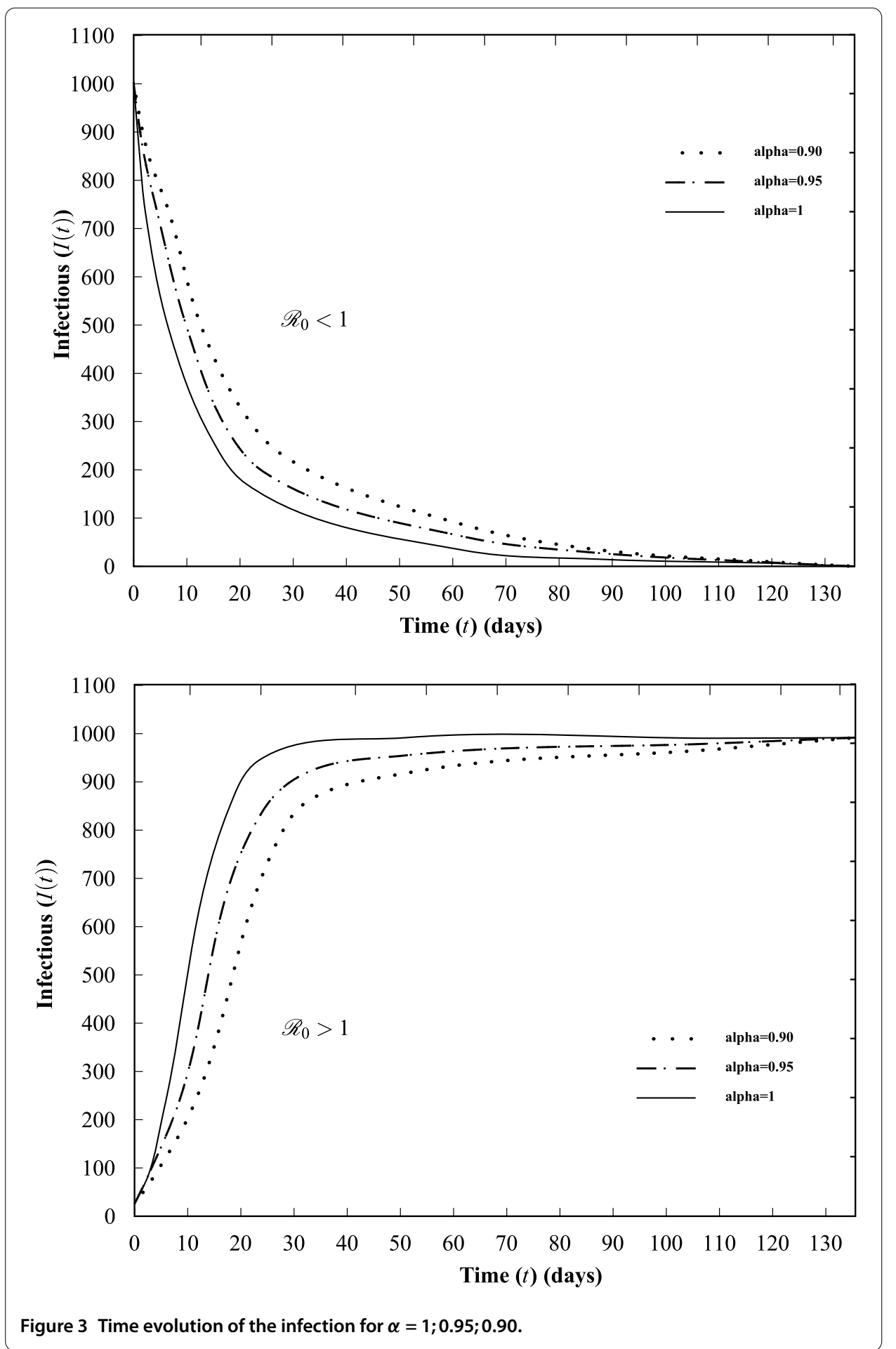




\section{Numerical simulations}

We consider, for reasons of simplicity, the nonlinear incidence function $g(I)=\frac{I^{p}}{1+\kappa I^{q}}$ and take $p=1$ and $\kappa=0$ to obtain $g(I)=I$. Thus, we find that the function $h$ defined in (9) becomes

$$
h(I)=1-\frac{I d}{b} .
$$

If $\frac{\beta g^{\prime}(0) b}{d(\gamma+d)}=\frac{\beta b}{d(\gamma+d)}<1$ then, according to Theorem 4.2, the disease free equilibrium of (6) is asymptotically stable.

If $\frac{\beta b}{d(\gamma+d)}>1$ then $\frac{d(\gamma+d)}{\beta b}<1=h(0)$ and $h^{\prime}(I)=-\frac{d}{b}<0$. Hence, according to Theorem 4.1, there is one endemic equilibrium point.

To provide a numerical simulations of the monotonic behavior of $I(t)$ and bring out those equilibrium points, with different values of $\alpha$, we use the implementation code of the predictor-corrector PECE method of Adams-Bashforth-Moulton type described in [20]. The time evolution of $I(t)$ is given in Figure 3. We note that, comparing to the system of differential equations with first-order derivative, there is a similar evolution of the infection for close values of $\alpha(0 \leq \alpha<1)$.

\section{Conclusion and possible future analysis}

Making use of the mean value theorem extended to fractional calculus [16], we have provided some remarkable and useful properties of a fractional Kermack-McKendrick epidemic model with nonlinear incidence function. We have shown that the existence of equilibrium points and the stability of the disease free equilibrium both depend on the nonlinear incidence function and we provide the expression of the basic reproduction number for the fractional model. The results obtained here are seen as the extension of the previous works with the inclusion of the derivative of order less than unity and a nonlinear incidence. Numerical simulations show the monotonic behavior of the infection $I(t)$ and comparing to the dynamics with first-order derivative, we note a similar evolution for close values of $\alpha(0 \leq \alpha<1)$. This may therefore help analyze the stability of the endemic equilibrium point for the model and also help to investigate more complex fractional epidemic models.

Competing interests

The authors declare that they have no competing interests.

\section{Authors' contributions}

All authors contributed equally to the manuscript and read and approved the final manuscript.

Received: 3 June 2014 Accepted: 17 October 2014 Published: 30 Oct 2014

References

1. Kermack, WO, McKendrick, AG: A contribution to mathematical theory of epidemics. Proc. R. Soc. Lond. A 115, 700-721 (1927)

2. Kermack, WO, McKendrick, AG: Contributions to the mathematical theory of epidemics. Bull. Math. Biol. 53, 33-55 (1991)

3. Brockmann, D, Hufnagel, L: Front propagation in reaction-superdiffusion dynamics: taming Lévy flights with fluctuations. Phys. Rev. Lett. 98, 178301 (2007)

4. Pooseh, S, Rodrigues, HS, Torres, DFM: Fractional derivatives in dengue epidemics. In: Simos, TE, Psihoyios, G, Tsitouras, C, Anastassi, Z (eds.) Proceedings of the International Conference on Numerical Analysis and Applied Mathematics, pp. 739-742. Am. Inst. of Phys., Melville (2011)

5. Oldham, KB, Spanier, J: The Fractional Calculus. Academic Press, New York (1974)

6. Miller, KS, Ross, B: An Introduction to the Fractional Calculus and Fractional Differential Equations. Wiley, New York (1993) 
7. Podlubny, I: Fractional Differential Equations. Academic Press, New York (1999)

8. Kilbas, AA, Srivastava, HM, Trujillo, JJ: Theory and Applications of Fractional Differential Equations. Elsevier, Amsterdam (2006)

9. Boto, JP: Review on fractional derivatives. CMAF manuscript (2009)

10. Samko, SG, Kilbas, AA, Marichev, OI: Fractional Integrals and Derivatives. CRC Press, Boca Raton (1993)

11. Baleanu, D, Diethelm, K, Scalas, E, Trujillo, JJ: Fractional Calculus: Models and Numerical Methods. World Scientific, Singapore (2012)

12. Atangana, $A, B$ Botha, JF: A generalized groundwater flow equation using the concept of variable order derivative. Bound. Value Probl. 2013, 53 (2013). doi:10.1186/1687-2770-2013-53

13. Diethelm, K: The Analysis of Fractional Differential Equations. Springer, Berlin (2010)

14. Doungmo Goufo, EF, Maritz, R, Mugisha, S: Existence results for a Marchaud fractional, nonlocal and randomly position structured fragmentation model. Math. Probl. Eng. 2014, Article ID 361234 (2014). doi:10.1155/2014/361234

15. Caputo, M: Linear models of dissipation whose $Q$ is almost frequency independent. J. R. Aust. Hist. Soc. 13(2), 529-539 (1967)

16. Odibat, ZM, Shawagfeh, NT: Generalized Taylor's formula. Appl. Math. Comput. 186, 286-293 (2007)

17. Liu, WM, Hethcote, HW, Levin, SA: Dynamical behavior of epidemiological models with nonlinear incidence rates. J. Math. Biol. 25, 359-380 (1987)

18. Hethcote, HW, van den Driessche, P: Some epidemiological models with nonlinear incidence. J. Math. Biol. 29 271-287 (1991)

19. Matignon, D: Stability results for fractional differential equations with applications to control processing. In: Computational Engineering in Systems Applications (Lille, France), vol. 2, pp. 963-968 (1996)

20. Diethelm, K, Ford, NJ, Freed, AD, Luchko, Y: Algorithms for the fractional calculus: a selection of numerical methods. Comput. Methods Appl. Mech. Eng. 194, 743-773 (2005)

10.1186/1687-1847-2014-278

Cite this article as: Doungmo Goufo et al: Some properties of the Kermack-McKendrick epidemic model with fractional derivative and nonlinear incidence. Advances in Difference Equations 2014, 2014:278

\section{Submit your manuscript to a SpringerOpen ${ }^{\circ}$ journal and benefit from:}

- Convenient online submission

Rigorous peer review

- Immediate publication on acceptance

Open access: articles freely available online

- High visibility within the field

- Retaining the copyright to your article 